\title{
On star formation rates in dwarf galaxies
}

\author{
P. M. Weilbacher and U. Fritze-v.Alvensleben \\ Universitäts-Sternwarte, Geismarlandstr. 11, 37083 Göttingen, Germany \\ Received 7 May 2001 / Accepted 16 May 2001

\begin{abstract}
We present evolutionary synthesis models of starbursts on top of old stellar populations to investigate in detailed time evolution the relation between $\mathrm{H}_{\alpha}$ luminosity and star formation rate (SFR). The models show that several effects have an impact on the ratio between $L\left(\mathrm{H}_{\alpha}\right)$ and SFR. Metallicity different from solar abundance, a time delay between star formation and maximum $\mathrm{H}_{\alpha}$-luminosity, and a varying stellar initial mass function give rise to strong variations in the ratio of $\mathrm{H}_{\alpha}$ luminosity to SFR and can cause large errors in the determination of the SFR when employing well-known calibrations. When studying star-bursting dwarf galaxies, and sub-galactic fragments at high redshift, which show SFR fluctuating on short timescales, these effects can add up to errors of two orders of magnitude compared with the calibrations. To accurately determine the true current SFR additional data in combination with models for the spectral energy distribution are needed.
\end{abstract}

Key words. galaxies: starbursts - galaxies: dwarf - galaxies: evolution - galaxies: fundamental parameters

\section{Introduction}

The star formation rate (SFR) is one of the basic properties of galaxies. It can be derived in different wavelength regimes (UV, optical, FIR) using empirical calibrations obtained from well studied samples of galaxy types, like spirals, irregulars, starbursts, or luminous IR galaxies. One of the most popular methods to derive SFRs from optical observations is by measuring the Balmer line fluxes, which are very sensitive to the HII regions surrounding massive young stars and therefore give a good measure of the very recent, shortlived, or ongoing SFR. When employing $\mathrm{H}_{\alpha}$ one generally takes one of the simple linear calibrations as e.g. obtained by Hunter \& Gallagher (1986, hereafter HG86) or Kennicutt et al. (1994, KTC94) from observations and modeling of dwarf irregular and spiral galaxies, respectively. The calibrations are used in the form ${ }^{1}$

$L\left(\mathrm{H}_{\alpha}\right)\left[\operatorname{erg~s}^{-1}\right]=\mathcal{V}^{2} \cdot \mathrm{SFR}\left[M_{\odot} \mathrm{yr}^{-1}\right]$,

where the $\mathcal{V}^{2}$-factor is $1.41 \times 10^{41}(\mathrm{HG} 86)$ or $1.26 \times 10^{41}$ (KTC94, Kennicutt 1998) for a Salpeter IMF in the mass range of 0.1 to $100 M_{\odot}$.

These $L\left(\mathrm{H}_{\alpha}\right)$-SFR calibrations were derived for "normal" galaxies with modest star formation rates. For

Send offprint requests to: P. Weilbacher,

e-mail: weilbach@uni-sw.gwdg.de

1 The name of the variable $\mathcal{V}^{2}=L\left(\mathrm{H}_{\alpha}\right) / \mathrm{SFR}$ is deduced from the fact, that the unit of $\mathcal{V}^{2}$ actually is a squared velocity. systems which behave differently from the ones for which the calibrations were derived, the application of this method may not be appropriate. The validity of the SFRdetermination in star-bursting galaxies, like e.g. blue compact dwarfs (BCDs), where these relations are frequently used, has never been shown.

In fact, as the strongest output of ionizing photons is related to the most luminous, i.e. giant or supergiant phases of the ionizing stars, there may be a small time delay between abrupt changes in the SFR and the corresponding changes in the $\mathrm{H}_{\alpha}$ flux. Leitherer et al. (1995) showed that such a time dependence exists for stellar features in the UV. For systems with SF fluctuations on short timescales, the delay effect of the Balmer lines can also be important, e.g. for small scale systems like dwarf galaxies, where star burst durations are usually assumed to be of the order of a dynamical timescale, i.e. $10^{5}$ to $10^{6}$ years. SFR fluctuating strongly on short timescales may also have taken place in sub-galactic fragments before merging together to hierarchically build up today's galaxies (Glazebrook et al. 1999).

We will show that indeed one may make very large errors when blindly applying the usual calibrations to small systems. We first present details on our model in Sect. 2. We then describe different effects that can affect the calibrations for $L\left(\mathrm{H}_{\alpha}\right)$ in terms of SFR, namely metallicity (Sect. 3), short burst durations (Sect. 4), and changes in the IMF (Sect. 5). We finally summarize our results in Sect. 6 . 


\section{Model description}

We use our evolutionary synthesis code specifically adapted to the modeling of starbursts in dwarf galaxies (Krüger et al. 1995; Weilbacher et al. 2000). It includes specific modeling of gaseous emission lines and continuum based on the Lyman continuum photons emitted by hot young stars.

We use the current Geneva stellar tracks (see Lejeune \& Schaerer 2001, for a recent compilation) in the metallicity range from $Z_{\odot} / 20$ to $2 Z_{\odot}$. To be compatible with HG86 and KTC94 we use the Salpeter IMF (Salpeter $1955)$ in the range of 0.15 to 85 or $120 M_{\odot}$, as given by the tracks. We use the recently revised Lyman continuum photon emission rates as given by Schaerer \& de Koter (1997), who account for non-LTE effects, line blanketing, stellar winds, and the new temperature and gravity calibrations by Vacca et al. (1996). To derive the $\mathrm{H}_{\alpha}$ luminosity, we sum up the emerging Lyman continuum photon emission $N\left(\mathrm{H}^{0}\right)$ for each star, and convert it to a luminosity in $\mathrm{H}_{\alpha}$ using

$$
L\left(\mathrm{H}_{\alpha}\right)\left[\mathrm{erg} \mathrm{s}^{-1}\right]=1.36 \times 10^{-12} N\left(\mathrm{H}^{0}\right)\left[\mathrm{s}^{-1}\right] .
$$

We have updated our models using the emission line ratios for low metallicities observed by Izotov et al. (1997) and Izotov \& Thuan (1998) for a large sample of blue compact dwarfs (BCDs).

We present two types of one-zone models. The first represents a quiescent galaxy, where the SFR decreases slowly from the formation epoch with a timescale of 10 Gyrs. The other model was already discussed in detail by Weilbacher et al. (2000) in their interpretation of Tidal Dwarf Galaxy candidates, and can also be used to model blue compact dwarfs (BCDs, see Krüger et al. 1995). Here we put a starburst on top of the stellar population of the undisturbed model. It is assumed to reach its maximum SFR of $20 M_{\odot} \mathrm{yr}^{-1}$ after 10 Gyrs, and we vary the burst timescale $\tau_{\mathrm{B}}$ from $10^{5}$ to $10^{8}$ yrs to model all the range of burst durations from small dwarf galaxies to mergers of giant gasrich galaxies. We alternatively a bell shaped (Gaussian) burst or sharply rising and exponentially decreasing starburst. We assume a minimum SFR after the starburst of $0.1 M_{\odot} \mathrm{yr}^{-1}$.

\section{Effects of metallicity}

We successfully reproduce the calibrations of HG86 and KTC94 using our quiescent models using solar metallicity and upper mass limits of 120 and $85 M_{\odot}$, respectively. The values we derive for the four other metallicities are given in Table 1.

While for solar metallicity models the agreement with HG86's and KTC94's empirical calibrations is very good, it is also seen in Table 1 that both towards lower and higher metallicities the differences become significant. For subsolar metallicities as e.g. in BCDs $\left(\langle Z\rangle_{\mathrm{BCD}}=0.002 \pm\right.$ $0.001=1 / 10 Z_{\odot}$, Izotov \& Thuan 1998) the difference in $\mathcal{V}^{2}$ is found to be a factor of $\sim 3.5$. This is a result of low
Table 1. Calibrations for different metallicities $Z$.

\begin{tabular}{lr|cc}
\hline \multicolumn{1}{c|}{$Z$} & $\begin{array}{r}M_{\text {up }} \\
{\left[M_{\odot}\right]}\end{array}$ & $\begin{array}{c}\mathcal{V}^{2} \\
{\left[10^{41}\right.}\end{array}$ & $\left.\begin{array}{c}\mathcal{V}_{[\mathrm{O} \text { II s }}^{2} \\
M_{\odot} \mathrm{yr}^{-1}\end{array}\right]$ \\
\hline 0.001 & 85 & 4.221 & 1.923 \\
0.001 & 120 & 4.702 & 2.142 \\
0.004 & 85 & 3.105 & 1.944 \\
0.004 & 120 & 3.315 & 2.076 \\
0.008 & 85 & 2.073 & 2.182 \\
0.008 & 120 & 2.548 & 2.682 \\
0.020 & 85 & 1.229 & 1.293 \\
0.020 & 120 & 1.408 & 1.482 \\
0.040 & 85 & 0.902 & 0.949 \\
0.040 & 120 & 0.997 & 1.049 \\
\hline 0.020 (S86) & 120 & 0.917 & 0.965 \\
HG86 & 100 & 1.41 & - \\
KTC94 & 100 & 1.26 & - \\
\hline
\end{tabular}

metallicity stellar populations being both more luminous and hotter than a solar metallicity stellar population with the same IMF and mass limits.

Application of the empirical calibrations to estimate SFRs from $\mathrm{H}_{\alpha}$ luminosities in low metallicity dwarf galaxies hence can yield SFRs overestimated by a factor $\gtrsim 3$ due to metallicity effects. We also give in Table 1 the $\mathcal{V}^{2}$-value obtained in our solar metallicity model using the IMF of Scalo (1986) instead of Salpeter's. Due to the smaller number of high mass stars in case of a Scalo-IMF, the same SFR produces an $\mathrm{H}_{\alpha}$ luminosity that is lower by $35 \%$.

All other hydrogen lines, as e.g. $\mathrm{L}_{\alpha}$ or $\mathrm{Br}_{\gamma}$, which can also be used to estimate SFRs, and their respective calibration factors can easily be computed from our values for $\mathrm{H}_{\alpha}$ and Eq. (1).

At higher redshift, the [O II $] 3727$ line is frequently used to derive SFRs, as e.g. for Lyman Break galaxies. This line involves a direct metallicity dependence in addition to the one inherent in the hydrogen lines which is due to differences in temperature and luminosity of stellar populations at various metallicities. Since high redshift starforming objects and, in particular, sub-galactic fragments both have low metallicities and possibly strongly fluctuating SFRs, care is needed to derive SFRs from $L([\mathrm{O} \mathrm{II}])$. We present the values of the $\mathcal{V}_{[\mathrm{O} \text { II }}^{2}$-factor in Col. 4 of Table 1 for the $[\mathrm{O}$ II] 3727 line, where the according linear relation with SFR is

$L([\mathrm{O} \mathrm{II}])\left[\mathrm{erg} \mathrm{s}^{-1}\right]=\mathcal{V}_{[\mathrm{O} \mathrm{II}]}^{2} \cdot \mathrm{SFR}\left[M_{\odot} \mathrm{yr}^{-1}\right]$.

\section{Short starbursts}

In Fig. 1 we show the time evolution of $L\left(\mathrm{H}_{\alpha}\right)$ for rapidly rising (instantaneous) starbursts with short timescales $\left(\tau_{\mathrm{B}}=10^{6} \mathrm{yr}\right)$ for five metallicities. All models have their maximum SFR at a time of 10.0 Gyrs. We note two effects: for lower metallicity $Z$ the maximum $\mathrm{H}_{\alpha}$ 


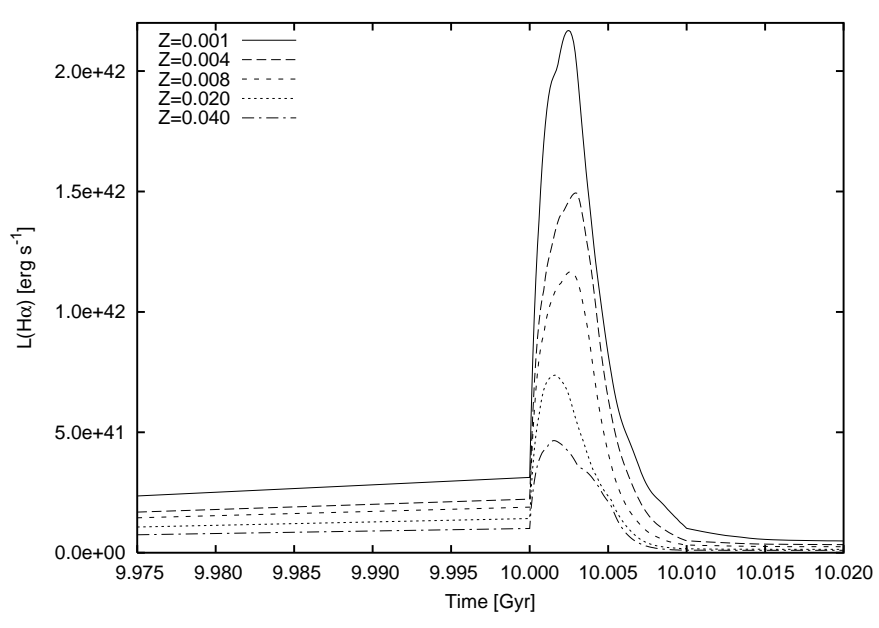

Fig. 1. $L\left(\mathrm{H}_{\alpha}\right)$ over time for instantaneous starbursts with short timescale of $\tau_{\mathrm{B}}=10^{6} \mathrm{yr}$.

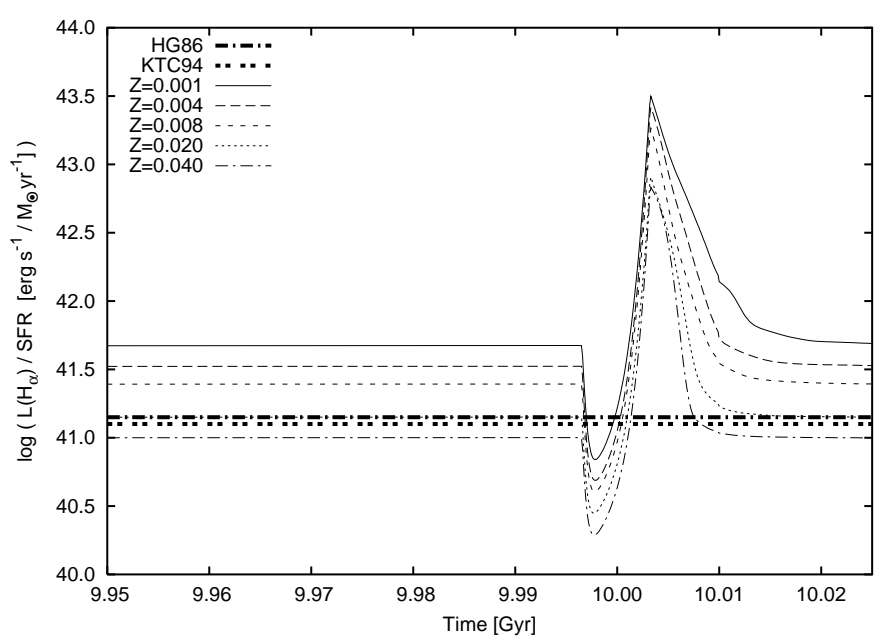

Fig. 2. $\mathcal{V}^{2}=L\left(\mathrm{H}_{\alpha}\right) /$ SFR over time for Gaussian bursts with short timescale of $\tau_{\mathrm{B}}=10^{6} \mathrm{yr}$.

luminosity is higher than for high metallicity, e.g. by a factor of 4.6 in case of $Z=0.001$ as compared with $Z=0.040$. This is due to the higher temperatures of the low metallicity stars, which more efficiently ionize the interstellar medium. It is also apparent that there is a delay of the maximum in the $\mathrm{H}_{\alpha}$ luminosity with regard to the maximum of the SFR. The offset between maximum SFR and maximum $L\left(\mathrm{H}_{\alpha}\right)$ is higher for lower metallicities, $2.9 \mathrm{Myrs}$ for $Z=0.004$ vs. $1.5 \mathrm{Myrs}$ for $Z=0.040$. This again is a result of the higher temperatures and hence the ionizing power of low metallicity stars. At low metallicity stars of lower mass and longer main sequence lifetimes contribute to the Lyman continuum emission. Since those take longer to reach their maximum Lyman continuum emission rates during their supergiant phase the delay becomes slightly longer than in the solar metallicity case.

In Fig. 2 we plot the ratio between $L\left(\mathrm{H}_{\alpha}\right)$ and the SFR of our models as a function of time for Gauss-shaped bursts with a short timescale of $\tau_{\mathrm{B}}=10^{6} \mathrm{yr}$. Before and after the starburst the empirical linear calibrations agree well with the ratio seen in our solar metallicity model.

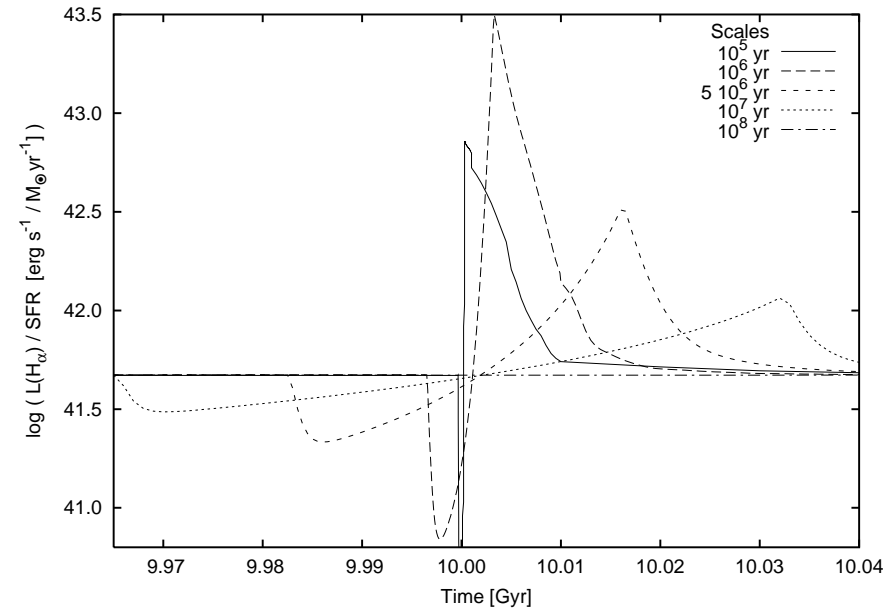

Fig. 3. $\mathcal{V}^{2}=L\left(\mathrm{H}_{\alpha}\right) /$ SFR over time for Gaussian bursts with different timescales and $Z=0.001$.

With the onset of the burst the SFR rises faster than $L\left(\mathrm{H}_{\alpha}\right)$ due to the delay in the maximum Lyman continuum photon production shown before in Fig. 1. Therefore the $\mathcal{V}^{2}$-factor first decreases. After its minimum $\mathcal{V}^{2}$ increases, because $L\left(\mathrm{H}_{\alpha}\right)$ continues to rise until the ionizing stars have reached their supergiant phase. When the number of Lyman continuum photons and therefore $L\left(\mathrm{H}_{\alpha}\right)$ become maximal, the SFR has already decreased from its maximum value by a factor of $\sim 3$. After its maximum $L\left(\mathrm{H}_{\alpha}\right)$ declines as the death rate of $\mathrm{O}$ stars is no longer compensated by SF. In this phase, however, the SFR decreases even faster than the $\mathrm{H}_{\alpha}$ flux, and $\mathcal{V}^{2}$ continues to increase. The sharp peak visible near 10.003 Gyrs, after which $\mathcal{V}^{2}$ abruptly decreases, is a result of the constant minimum SFR of our models after the burst. If the SFR after the burst would go to zero, $\mathcal{V}^{2}$ would diverge. The minimum SFR, which starts near 10.003 Gyrs, then acts to slowly bring down $\mathcal{V}^{2}$ to values in agreement with those in Table 1 . This time sequence during the starburst and the interplay between $L\left(\mathrm{H}_{\alpha}\right)$ and SFR can also be seen in Fig. 4 below.

It is obvious from Fig. 2 that for bursts with $\tau_{\mathrm{B}}=$ $10^{6} \mathrm{yr}$ strong discrepancies from the calibrations are seen for all metallicities, strongest at the lowest metal abundance, amounting to a difference of nearly two orders of magnitude as compared to the calibrations, even for solar metallicity models.

In Fig. 3 we show the evolution of the $\mathcal{V}^{2}$-factor with time for five Gaussian starburst models with different burst timescales for our lowest metallicity $Z=0.001$. With increasing burst duration, the maximum is shifted towards later times. It is strongest for bursts with $\tau_{\mathrm{B}}=$ $10^{6} \mathrm{yr}$ (which are shown in Fig. 2). This is a result of the convolution of the Gauss-shaped increase of the burst-SFR with the delay in maximum $\mathrm{H}_{\alpha}$ emission due to the most massive and most luminous (= giant) stars. The decline after the sharply peaked maximum is an effect of the onset of the minimum SFR as discussed above for Fig. 2. The time evolution of $\mathcal{V}^{2}$ significantly depends on the burst 


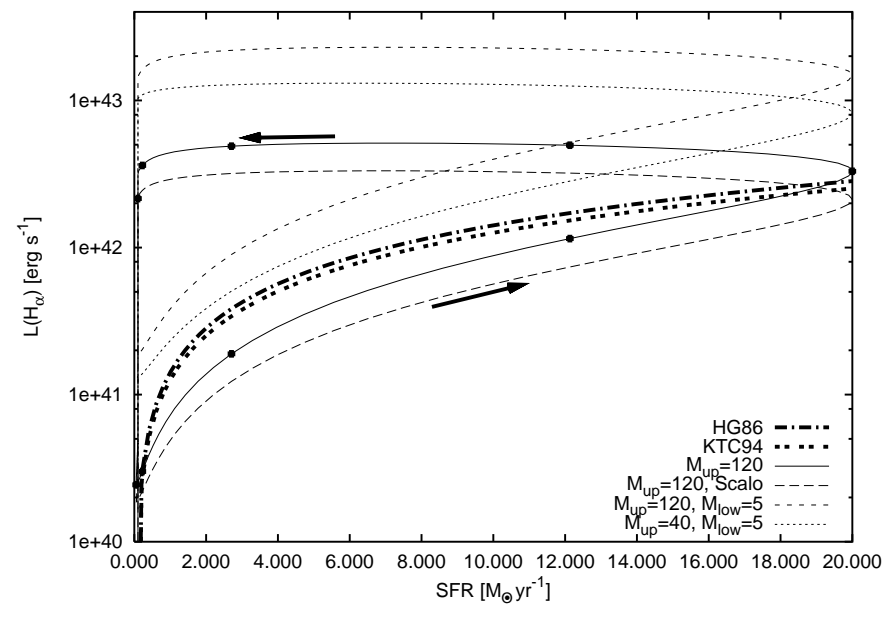

Fig. 4. $L\left(\mathrm{H}_{\alpha}\right)$ over SFR for a bursting dwarf galaxy with metallicity $Z=0.001$ and different IMF cuts.

duration. For the longest burst with $\tau_{\mathrm{B}}=10^{8} \mathrm{yr}$ the slow change in the SFR of the burst causes the delay effect to have negligible impact on $\mathcal{V}^{2}$.

In conclusion we have shown that the SFR derived from $\mathrm{H}_{\alpha}$ during or shortly after short bursts can be wrong by factors $\gg 10$. Averaged over the entire burst duration, however, values for $\mathcal{V}^{2}$ agree well with those from Table 1 for different metallicities. This applies to statistical analyses of samples of $\mathrm{H}$ II galaxies.

\section{Effects of the IMF}

Kennicutt et al. (1994) have already investigated different $\mathcal{V}^{2}$-factors with different IMFs with identical mass cutoffs and found differences of an order of magnitude between IMFs they used. Leitherer \& Heckman (1995) also presented properties like number of O stars, $N\left(\mathrm{H}^{0}\right)$, and $E W\left(\mathrm{H}_{\alpha}\right)$ etc. for instantaneous and continuous SF models with different IMFs. Here we want to show the effect of various IMFs on the time delay discussed above.

Figure 4 shows the luminosity $L\left(\mathrm{H}_{\alpha}\right)$ plotted over the SFR of the model galaxy, while it experiences its starburst with a timescale of $\tau_{\mathrm{B}}=10^{6} \mathrm{yr}$. The calibrations of HG86 and KTC94 are plotted for reference. To indicate the evolution in time our "standard model" with $M_{\text {up }}=120 M_{\odot}$ has additional dots each $10^{6}$ yrs during the burst; the arrows show the direction of the loops in this diagram.

It is obvious that the calibrations do not represent the real SFR very well. $L\left(\mathrm{H}_{\alpha}\right)$ at the peak SFR does fit very well with the calibrations for both models with Salpeter and Scalo IMF and a high mass cut at $M_{\text {up }}=120 M_{\odot}$. But generally our models show that for an observed value of $L\left(\mathrm{H}_{\alpha}\right)$ there is an ambiguity between two values of the SFR. E.g. for $L\left(\mathrm{H}_{\alpha}\right)=10^{42} \mathrm{erg} \mathrm{s}^{-1}$ the calibrations give a SFR of $7 \ldots 8 M_{\odot} \mathrm{yr}^{-1}$, while one has to choose between SFRs of $\sim 0$ and $\sim 15 M_{\odot} \mathrm{yr}^{-1}$ from our model with Salpeter IMF, and one needs additional data to determine the true current SFR.
The evolution of the models after the starburst shows that for low SFRs (lower as one would expect from the calibrations) a galaxy could show high $\mathrm{H}_{\alpha}$ luminosity for quite some time $\left(\sim 3 \times 10^{6} \mathrm{yr}\right)$. For the two models with Salpeter IMF and lower mass cuts at $M_{\text {low }}=5 M_{\odot}$ this effect is even more extreme. $L\left(\mathrm{H}_{\alpha}\right)$ at the peak SFR is already underestimated by the calibrations by factors of 3 and 5, respectively, for high mass cutoffs of $M_{\mathrm{up}}=40$ and $120 M_{\odot}$. For a given $\mathrm{H}_{\alpha}$-luminosity the calibrations yield a SFR too high by at least one order of magnitude when compared to the values from our models.

\section{Conclusions}

When observing small scale star-forming entities like dwarf galaxies or sub-galactic fragments, where dynamical timescales and hence burst durations may typically be of the order of $10^{6}$ yrs or less, one should be aware that the currently used calibrations on the basis of $\mathrm{H}_{\alpha}$ luminosities may yield SFRs with large errors. These calibrations were determined from larger low-level star-forming systems (where they work very well), and when applying them to star-bursting dwarf galaxies, errors of factors 3 to 100 may affect this determination of the SFR. The ratio of $\mathrm{H}_{\alpha}$-luminosity to SFR depends on the metallicity of the object, and the age and the duration of the starburst.

To more accurately determine the true current SFR of a (star-bursting) dwarf galaxy additional information about the spectral energy distribution (SED) is needed. Observations in at least three optical/NIR filters or a spectrum with sufficient wavelength coverage to determine the slope of the SED could be used in comparison with models that include gaseous emission to eliminate ambiguities in the relation of the $\mathrm{H}_{\alpha}$ luminosity to the SFR.

Acknowledgements. We thank our anonymous referee for a very prompt and helpful report. PMW is partially supported by Deutsche Forschungsgemeinschaft (DFG Grant FR 916/6-1).

\section{References}

Glazebrook, K., Blake, C., Economou, F., Lilly, S., \& Colless, M. 1999, MNRAS, 306, 843

Hunter, D. A., \& Gallagher, J. S. 1986, PASP, 98, 5

Izotov, Y., \& Thuan, T. 1998, ApJ, 500, 188

Izotov, Y., Thuan, T., \& Lipovetsky, V. 1997, ApJS, 108, 1

Kennicutt, R. C. 1998, ARA\&A, 36, 189

Kennicutt, R. C., Tamblyn, P., \& Congdon, C. E. 1994, ApJ, 435,22

Krüger, H., Fritze-v.Alvensleben, U., \& Loose, H.-H. 1995, A\&A, 303, 41

Leitherer, C., \& Heckman, T. 1995, ApJS, 96, 9

Leitherer, C., Robert, C., \& Heckman, T. 1995, ApJS, 99, 173

Lejeune, T., \& Schaerer, D. 2001, A\&A, 366, 538

Salpeter, E. E. 1955, ApJ, 121, 161

Scalo, J. 1986, Fund. Cosmic Phys., 11, 1

Schaerer, D., \& de Koter, A. 1997, A\&A, 322, 598

Vacca, W. D., Garmany, C. D., \& Shull, J. M. 1996, ApJ, 460, 914

Weilbacher, P., Duc, P.-A., Fritze-v.Alvensleben, U., Martin, P., \& Fricke, K. 2000, A\&A, 358, 819 